\title{
Hole Pockets in the Doped 2D Hubbard Model
}

\author{
Adriana Moreo and Daniel Duffy \\ Department of Physics, National High Magnetic Field Lab and MARTECH, Florida State \\ University, Tallahassee, FL 32306, USA
}

(June 20, 2021)

\begin{abstract}
The electronic momentum distribution $\mathrm{n}(\mathbf{k})$ of the two dimensional Hubbard model is studied for different values of the coupling $\mathrm{U} / \mathrm{t}$, electronic density $\langle\mathrm{n}\rangle$, and temperature, using quantum Monte Carlo techniques. A detailed analysis of the data on $8 \times 8$ clusters shows that features consistent with hole pockets at momenta $\mathbf{k}=\left( \pm \frac{\pi}{2}, \pm \frac{\pi}{2}\right)$ appear as the system is doped away from half-filling. Our results are consistent with recent experimental data for the cuprates discussed by Aebi et al. (Phys. Rev. Lett. 72, 2757 (1994)). In the range of couplings studied, the depth of the pockets is maximum at $\langle\mathrm{n}\rangle \approx 0.9$, and it increases with decreasing temperature. The apparent absence of hole pockets in previous numerical studies of this model is explained.
\end{abstract}

Typeset using REVTEX 
One of the simplest models often used to describe the physics of the high temperature cuprate superconductors is defined by the two dimensional (2D) Hubbard Hamiltonian. Several normal state properties of these materials, such as antiferromagnetism, the behavior of the magnetic susceptibility with temperature and density, the optical conductivity and others, are qualitatively reproduced by this model. [1] Although superconductivity has not been observed in Monte Carlo simulations [2], self-consistent techniques suggest its existence at low temperatures in the $\mathrm{d}_{\mathrm{x}^{2}-\mathrm{y}^{2}}$ channel. [3] In order to understand the pairing mechanism leading to such superconducting instability it is important to study the shape of the Fermi surface in this model, and compare it against angle-resolved photoemission experiments for the cuprates. Theories based on antiferromagnetism (AF) explain in a natural way the apparent d-wave symmetry of the condensate, but they also suggest the existence of "hole pockets" in $\mathrm{n}(\mathbf{k})$. [⿴囗 에 Since for some time evidence of a large electron-like Fermi surface has been reported in experiments [5], ideas based on AF have been questioned. Moreover, in agreement with these experiments, theoretical studies of the Hubbard [6] and t-J Hamiltonians [7], reported electron-like Fermi surfaces very similar in shape to the non-interacting case, even at low hole densities where antiferromagnetic correlations should still be strong in the system. Possible many-body effects have been invoked to explain such a behavior.

However, on the experimental side, these results have been recently challenged by Aebi et al. 8] Using a photoemission technique that allows the mapping of the whole Fermi surface, these authors have shown the existence of hole pockets in their data for Bi2212 consistent with a rigid band filling of the hole states predicted by antiferromagnetically based meanfield approximations. [4] In parallel to the experimental results, recent theoretical arguments by Dagotto et al. 99 conjectured that the large Fermi surface of the 2D Hubbard model observed at finite temperature can be made compatible with the existence of hole pockets. Their reasoning is that unless the temperature of the simulation is smaller than the energy difference of holes states at momenta $\mathbf{k}=\left( \pm \frac{\pi}{2}, \pm \frac{\pi}{2}\right)$ and $(0, \pi),(\pi, 0)$ (which they found to be very small at $\mathrm{U} / \mathrm{t}=10$ ), the hole pocket effect is washed out for thermodynamical reasons. These authors suggested that studies at stronger couplings, lower temperatures and larger 
lattices should provide evidence for the existence of hole pockets.

Motivated by these challenging experimental and theoretical ideas, in this paper a careful analysis of Quantum Monte Carlo data for the 2D Hubbard model is discussed. We conclude that numerical evidence indeed supports the existence of hole pockets at $\mathbf{k}=\left( \pm \frac{\pi}{2}, \pm \frac{\pi}{2}\right)$ at low hole density. Thus, our results reopen the possibility that the normal state properties of the high temperature superconductors at half-filling can be qualitatively approximated by a band filling of the states obtained in the mean-field spin-density-wave approximation. The Hubbard Hamiltonian is given by

$$
\mathrm{H}=-\mathrm{t} \sum_{<\mathbf{i} \mathbf{j}>, \sigma}\left(\mathrm{c}_{\mathbf{i}, \sigma}^{\dagger} \mathrm{c}_{\mathbf{j}, \sigma}+\text { h.c. }\right)+\mathrm{U} \sum_{\mathbf{i}}\left(\mathrm{n}_{\mathbf{i} \uparrow}-1 / 2\right)\left(\mathrm{n}_{\mathbf{i} \downarrow}-1 / 2\right)+\mu \sum_{\mathbf{i}, \sigma} \mathrm{n}_{\mathbf{i}, \sigma},
$$

where $\mathrm{c}_{\mathbf{i}, \sigma}^{\dagger}$ creates an electron at site $\mathbf{i}$ with spin projection $\sigma, \mathrm{n}_{\mathbf{i}, \sigma}$ is the number operator, and the sum $\langle\mathbf{i j}\rangle$ runs over pairs of nearest neighbor lattice sites. $U$ is the on site coulombic repulsion, the hopping amplitude, and $\mu$ is the chemical potential. This Hamiltonian will be analyzed using standard Quantum Monte Carlo techniques. [10] Clusters with up to $8 \times 8$ sites will be studied varying the temperature $\mathrm{T}$, filling $\langle\mathrm{n}\rangle$, and coupling $\mathrm{U} / \mathrm{t}$. The notorious sign-problem prevents us from working at very low temperatures and large couplings. Those used in this paper correspond to the lowest $\mathrm{T}$ and largest $\mathrm{U} / \mathrm{t}$ that can be reliably reached with confidence using the QMC algorithm. [11] We will show below that they are enough to observe hole pockets in the Hubbard model.

The first issue to analyze is whether antiferromagnetic correlations are strong in the system away from half-filling, since this is a necessary condition for the existence of hole pockets induced by AF. In Fig.1-a, the spin-spin correlation function $\mathrm{C}(\mathbf{r})=(-1)^{|r|}\left\langle\mathrm{S}_{\mathbf{i}}^{z} \mathrm{~S}_{\mathbf{i}+\mathbf{r}}^{\mathrm{z}}\right\rangle$ is presented for $\mathrm{U} / \mathrm{t}=6$ on an $8 \times 8$ lattice at $\mathrm{T}=\mathrm{t} / 4$. At half-filling, $\langle\mathrm{n}\rangle=1$, (filled circles) the correlations are strong and remain finite even at the largest possible distance clearly indicating the expected antiferromagnetic long range order (LRO) in the system. More interesting for our analysis is that at density $\langle\mathrm{n}\rangle=0.9$ (open circles) where antiferromagnetic LRO does not exist, it is clear that the spin correlations are strong up to a distance of about 3 to 4 lattice spacings. Increasing further the hole density to $\langle\mathrm{n}\rangle=0.75$ (triangles), 
antiferromagnetism is further suppressed but it remains strong between nearest-neighbors spin showing that the moments are still well-formed even at this fairly large hole doping. When the coupling is reduced to the value that has been more widely analyzed by QMC techniques namely $\mathrm{U} / \mathrm{t}=4$, a qualitatively similar behavior is found at the same temperature. The spin correlations at this coupling (shown in Fig.1-b) are less developed, but LRO is still observed at half-filling. Working at $\langle\mathrm{n}\rangle=0.9$ short range antiferromagnetism is also robust as it occurs at higher couplings. Decreasing the temperature to $\mathrm{T}=\mathrm{t} / 6$ (shown in Fig.1-c) the spin correlations at $\mathrm{U} / \mathrm{t}=4$ become as strong as for $\mathrm{U} / \mathrm{t}=6$ at $\mathrm{T}=\mathrm{t} / 4$. Then, Figs.1-a,b,c have shown that at a realistic density of $\langle\mathrm{n}\rangle=0.9$, the antiferromagnetic correlations are sufficiently developed that they may originate hole-pockets in the Fermi surface. Eventually, these pockets should evolve into a noninteracting-like Fermi surface as the electronic density is further decreased from half-filling.

Since the antiferromagnetic correlations are robust, then the natural question to discuss is why hole pockets have not been observed in previous studies (which were only carried out at $\mathrm{U} / \mathrm{t}=4$ ). To address this question let us concentrate on the analysis of the momentum distribution $\mathrm{n}(\mathbf{k})=\sum_{\sigma} \mathrm{c}_{\mathbf{k}, \sigma}^{\dagger} \mathrm{c}_{\mathbf{k}, \sigma}$, where $\mathrm{c}_{\mathbf{k}, \sigma}^{\dagger}=\sum_{\mathbf{j}} \mathrm{e}^{\mathbf{i k} . \mathbf{j}} \mathrm{c}_{\mathbf{j}, \sigma}^{\dagger}$. Note that $\mathrm{n}(\mathbf{k})$ has been previously measured [6:0] but in these and some other papers, the shape of the Fermi surface was determined by calculating the position of the momenta where $\mathrm{n}(\mathbf{k})=0.5$. In principle, this procedure is correct. However, using such a convention here we show that the pockets could be overlooked due to finite temperature and/or small lattice effects. To understand this point, in Fig.2-a $n(\mathbf{k})$ versus $\mathbf{k}$ is shown along the $\mathrm{k}_{\mathrm{x}}=\mathrm{k}_{\mathrm{y}}$ direction for $\mathrm{U} / \mathrm{t}=6$ at $\mathrm{T}=\mathrm{t} / 4$ on an $8 \times 8$ cluster and density $\langle\mathrm{n}\rangle=0.9$ (open squares). Comparing the interacting results with noninteracting $\mathrm{U} / \mathrm{t}=0$ data for the same parameters (filled squares) we observe that, in both cases, $\mathrm{n}(\mathbf{k})$ becomes 0.5 at approximately the same momentum. Repeating the calculation along other directions in momentum space, and if the $\mathrm{n}(\mathbf{k}) \approx 0.5$ criterion is still used, it is thus understandable why a Fermi surface resembling a non-interacting system was obtained in previous studies. However, this does not rule out the existence of pockets. The crosses shown in Fig.2-a correspond to spin density wave mean-field (SDW- 
MF) results. 四 In this approximation, an antiferromagnetic state is used which effectively produces a $2 \times 2$ unit cell. The mean-field Hamiltonian is diagonalized producing conduction and valence bands separated by the antiferromagnetic gap. The energy levels are given by $E_{k}= \pm \sqrt{\epsilon_{k}^{2}+\Delta^{2}}$, where $\epsilon_{k}=-2 t\left(\cos k_{x}+\cos k_{y}\right)$, and $\Delta$ is found using a self-consistent equation. At half-filling, the valence band is filled, and $\mathrm{n}(\mathrm{k})=\frac{1}{2}\left(1-\frac{\epsilon_{k}}{E_{k}}\right)$ is in very good agreement with the numerical data [12] as it is shown in Fig.2-b. Now, let us assume that a robust antiferromagnetism survives the introduction of hole doping, as it was suggested by the results of Fig.1-a,b,c. Quasiparticles are removed from the top of the valence band to mimic the presence of doping. $\mathrm{n}(\mathbf{k})$ now is given by,

$$
\mathrm{n}(\mathbf{k})=\frac{1}{2}\left(1-\frac{\epsilon_{\mathbf{k}}}{\mathrm{E}_{\mathbf{k}}}\right)\left(\frac{1}{\mathrm{e}^{-\beta\left(\left|\mathrm{E}_{\mathbf{k}}\right|-\mu\right)}+1}\right),
$$

where $\mu$ is selected such that the density is $\langle\mathrm{n}\rangle$. In Fig.2a-b the SDW mean-field results are compared against the Monte Carlo data. The agreement along the $\mathrm{k}_{\mathrm{x}}=\mathrm{k}_{\mathrm{y}}$ direction both at half-filling and at finite hole density is remarkable. We have explicitly checked that only at densities where the antiferromagnetic correlations become of one lattice spacing or less $(\langle\mathrm{n}\rangle \approx 0.75)$, the comparison between the numerical data and the SDW results deteriorates. Reducing further the density, the QMC data converges smoothly to a weakly interacting gas of electrons.

The agreement between a theory based on strong AF correlations and the numerical data is consistent with the strong AF correlations shown in Fig.1. However, the main issue addressed in this paper remains paradoxical, i.e. if the numerical data and the SDW approximation are in good agreement, why there is no hole pocket in the occupation number mean value of Fig.2-a? To understand this point, it is instructive to study the effects that high temperatures and a finite lattice produce in $n(\mathbf{k})$ in the mean-field approximation. In Fig.3-a we show the mean field $\mathrm{n}(\mathbf{k})$ along the $\mathrm{k}=\mathrm{k}_{\mathrm{x}}=\mathrm{k}_{\mathrm{y}}$ direction on a $20 \times 20$ lattice, for $\mathrm{U} / \mathrm{t}=6$ and $\langle\mathrm{n}\rangle=0.9$. At $\mathrm{T}=0$ there is a clear pocket-like feature at $\left(\frac{\pi}{2}, \frac{\pi}{2}\right)$, very different from that observed numerically in Fig.2a. However, as the temperature increases the pocket 
becomes less pronounced and for temperatures $\mathrm{T}=\mathrm{t} / 6$ and $\mathrm{t} / 4$, which are the values most commonly studied with Monte Carlo techniques, it has all but disappeared. In Fig.2-a the crosses are the mean-field results and it is clear that they are in much better agreement with the Monte Carlo data than the $\mathrm{U} / \mathrm{t}=0$ points. This shows that the existence of pockets along the $\mathrm{k}=\mathrm{k}_{\mathrm{x}}=\mathrm{k}_{\mathrm{y}}$ direction is a feature very difficult to see and quickly washed out by high temperature effects [13] due to the rapid change in $n(\mathbf{k})$ where the pockets are expected. In Fig.3-b, we show the region on the $\mathrm{U} / \mathrm{t}-\mathrm{T} / \mathrm{t}$ plane where a pocket, defined as a local minimum in the momentum distribution, is expected at $\left(\frac{\pi}{2}, \frac{\pi}{2}\right)$ according to the SDW mean-field results at $\langle\mathrm{n}\rangle=0.9$. In the same plot the crosses indicate the lowest temperatures that can be presently reached for each $\mathrm{U} / \mathrm{t}$ value with the $\mathrm{QMC}$ algorithm, the limitation being caused mainly by the well-known sign-problem. Clearly, all the crosses lie in a region where pockets are not observed in the mean-field approximation. Thus, the absence of a local minimum in our results of Fig.2-a are not incompatible with hole pockets, and it is caused by finite temperature and small lattice effects.

The most striking evidence showing that the interacting system is different from the non-interacting one is obtained analyzing the line from $\mathbf{k}=(0, \pi)$ to $(\pi, 0)$ (i.e. from $\mathrm{Y}$ to $\mathrm{X})$. The SDW-MF predicts a constant momentum distribution along $\cos k_{x}+\cos k_{y}=0$ even at finite coupling, but this is a spurious degeneracy of this simple approximation and thus it is not useful to contrast against the QMC data. The noninteracting system $\mathrm{U} / \mathrm{t}=0$, also has $\mathrm{n}(\mathbf{k})$ constant along this line at any density and temperature, and it is given by

$$
\mathrm{n}(\mathbf{k})=\frac{1}{\mathrm{e}^{\beta\left(\epsilon_{\mathbf{k}}-\mu\right)}+1}=\frac{1}{\mathrm{e}^{-\beta \mu}+1} .
$$

At half-filling $\mu=0$ and $\mathrm{n}(\mathbf{k})$ along this line is 0.5 . This symmetry allows us to study pockets in the interacting system by looking for a reduction along the X-Y line of the momentum distribution as the coupling is increased. If $\mathrm{n}\left(\frac{\pi}{2}, \frac{\pi}{2}\right)$ is the smallest along this line, it would be an evidence in favor of hole pockets. In Fig.4-a $n(\mathbf{k})$ vs $\mathbf{k}$ along the $(\pi, 0)-(0, \pi)$ line is presented, using an $8 \times 8$ cluster, $\mathrm{U} / \mathrm{t}=6$, and $\mathrm{T}=\mathrm{t} / 4$. In the half-filled case (filled circles $) \mathrm{n}(\mathbf{k})$ is constant even for an interacting system. However, at density $\langle\mathrm{n}\rangle=0.9$ 
(open circles) there is a clear minimum (pocket) at $\mathbf{k}=\left(\frac{\pi}{2}, \frac{\pi}{2}\right)$. The minimum lasts as long as antiferromagnetism is present in the system, i.e. at density $\langle n\rangle \leq 0.75$ or smaller the minima are no longer observed giving support to our interpretation of this feature as hole pockets caused by AF correlations.

In Fig.4-b, similar results are presented for $\mathrm{U} / \mathrm{t}=4$. Here the pockets are smaller than at $\mathrm{U} / \mathrm{t}=6$ because the $\mathrm{AF}$ order is not so strong but the qualitative behavior is the same. The maximum depth occurs again for $\langle n\rangle=0.9$. In Fig.4-c we show the pocket at several couplings $\mathrm{U} / \mathrm{t}$ ranging from 0 to 6 at a fixed density $\langle\mathrm{n}\rangle=0.9$. As the coupling increases the pocket becomes deeper which is intuitively understandable using the AF picture. Finally, in Fig.4-d the dependence with temperature at $\mathrm{U} / \mathrm{t}=4$ and $\langle\mathrm{n}\rangle=0.9$ is presented. As with the coupling dependence, the pocket becomes deeper as the temperature is lowered from $\mathrm{T}=\mathrm{t} / 2$ to $\mathrm{t} / 6$. [14] It is clear that the behavior of the pocket is correlated with the behavior of the magnetic correlations presented in Fig.1. In other words, when the spin correlations are strong, the pockets appear in the spectra. When the correlations increase, due to an increase in coupling or decrease in temperature, the pockets become deeper. But when the AF correlations are of the order of one lattice spacing, as for $\langle\mathrm{n}\rangle<0.75$, the pockets are no longer observed, and $\mathrm{n}(\mathbf{k})$ becomes constant along the $(\pi, 0),(0, \pi)$ direction as in the noninteracting case.

In this paper, we have presented evidence that in the 2D Hubbard model the strong antiferromagnetic fluctuations produce hole pockets at momenta $\mathbf{k}=\left( \pm \frac{\pi}{2}, \pm \frac{\pi}{2}\right)$. Monitoring the behavior of $\mathrm{n}(\mathbf{k})$ along the line $(\pi, 0),(0, \pi)$, where the electronic momentum distribution is constant in the noninteracting system, we have observed a minimum at $\left(\frac{\pi}{2}, \frac{\pi}{2}\right)$ compatible with the existence of a hole pocket. Along the $\mathrm{k}_{\mathrm{x}}=\mathrm{k}_{\mathrm{y}}$ direction no local minimum is numerically observed but by comparing our results to a SDW-MF approximation we have shown that this feature is due to finite temperature effects, as predicted by Dagotto et al. [9] For the values of $\mathrm{U} / \mathrm{t}$ here studied, i.e. 4 and 6 , the pockets exist for $\langle n\rangle>0.75$ and the maximum effect is observed at $\langle\mathrm{n}\rangle=0.9$. Larger couplings and lower temperatures would certainly increase the range of existence of these hole pockets. Thus, we have shown that the 
recent experiments of Aebi et al. are compatible with results obtained for the 2D Hubbard model giving support to theoretical descriptions of the cuprates based on antiferromagnetic correlations.

We thank E. Dagotto for useful conversations and suggestions. A. M. is supported by the Office of Naval Research under grant ONR N00014-93-0495. We thank SCRI and the Computer Center at FSU for providing us access to the Cray-YMP and ONR for giving us access to the CM5 connection machine. 


\section{REFERENCES}

[1] For a complete list of references see E. Dagotto, NHMFL preprint, Rev. Mod. Phys., July 1994.

[2] A. Moreo, Phys. Rev. B 45, 5059 (1992).

[3] C-H. Pao and N. E. Bickers, Phys. Rev. Lett. 72, 1870 (1994); P. Monthoux and D. Pines, Phys. Rev. Lett. 72, 1874 (1994).

[4] J.R. Schrieffer, X.G. Wen and S.C. Zhang, Phys. Rev. B 39, 11663 (1989).

[5] J.C. Campuzano, et al., Phys. Rev. Lett. 64, 2308 (1990) and Phys. Rev. B 43, 2788 (1991); Liu Rong, et al., Phys. Rev. B 45, 5614 (1992) and Phys. Rev. B 46, 11056 (1992).

[6] A. Moreo et al., Phys. Rev. B 41, 2313 (1990).

[7] W. Stephan and P. Horsch, Phys. Rev. Lett. 66, 2258 (1991); R.R.P. Singh and R.L. Glenister, Phys. Rev. B 46, 14313 (1992).

[8] P. Aebi et al., Phys. Rev. Lett. 72, 2757 (1994).

[9] E. Dagotto, A. Nazarenko and M. Boninsegni, to appear in Phys. Rev. Lett.

[10] R. Blankenbecler, D.J. Scalapino and R. Sugar, Phys. Rev. D 24, 2278 (1981).

[11] The data for $\mathrm{U} / \mathrm{t}=6$ were obtained using $\Delta \tau=0.0625$ while for the remaining values of $\mathrm{U} / \mathrm{t}$ we used $\Delta \tau=0.125$. The number of Monte Carlo iterations used depended on the filling. For each point we did at least two independent runs with 2000 warm ups and 8000 measurement sweeps.

[12] For values of $\mathrm{U} / \mathrm{t}$ smaller than 6 , i.e. for $\mathrm{U} / \mathrm{t}=4$, the mean field result gives a reasonable qualitative fit to the Monte Carlo data. Quantitative agreement is obtained by reducing $\Delta[6]$. 
[13] In small lattices, .i.e. $4 \times 4,8 \times 8$, not even at $\mathrm{T}=0$ the pockets are observed at the mean field level along the $\mathrm{k}_{\mathrm{x}}=\mathrm{k}_{\mathrm{y}}$ direction.

[14] We also studied couplings ranging from $\mathrm{U} / \mathrm{t}=0$ to 8 at $\mathrm{T}=\mathrm{t} / 3$ and we observed that at $\langle\mathrm{n}\rangle=0.9$ the depth of the pocket increased with $\mathrm{U} / \mathrm{t}$. However, the pocket is deeper for $\mathrm{U} / \mathrm{t}=6$ and $\mathrm{T}=\mathrm{t} / 4$ than for $\mathrm{U} / \mathrm{t}=8$ and $\mathrm{T}=\mathrm{t} / 3$. This can be understood by noting in Fig.2-b that the first case is closer to the pocket region than the second.

\section{Figure Captions}

1. (a) Spin-spin correlation $\mathrm{C}(\mathbf{r})=\left\langle\mathrm{S}_{\mathbf{i}}^{\mathrm{z}} \mathrm{S}_{\mathbf{i}+\mathbf{r}}^{\mathrm{z}}\right\rangle(-1)^{|\mathbf{r}|}$ for $\mathrm{U} / \mathrm{t}=6, \mathrm{~T}=\mathrm{t} / 4$ on an $8 \times 8$ lattice at different fillings. The error bars are of the size of the dots.

(b) Same as (a) but for $\mathrm{U} / \mathrm{t}=4$.

(c) Same as (b) but for $\mathrm{T}=\mathrm{t} / 6$.

2. (a) $\mathrm{n}(\mathbf{k})$ as a function of momentum along the $\mathrm{k}_{\mathrm{x}}=\mathrm{k}_{\mathrm{y}}$ diagonal direction on an $8 \times 8$ lattice for $\mathrm{U} / \mathrm{t}=6, \mathrm{~T}=\mathrm{t} / 4$ at $\langle\mathrm{n}\rangle=0.9$. The open squares are Monte Carlo results, the filled squares are $\mathrm{U} / \mathrm{t}=0$ results, and the crosses correspond to the SDW mean field approximation (see text).

(b) Same as (a) for $\langle\mathrm{n}\rangle=1.0$ (half-filling).

3. (a) Mean field values of $\mathrm{n}(\mathbf{k})$ vs momenta along the diagonal $\mathrm{k}_{\mathrm{x}}=\mathrm{k}_{\mathrm{y}}$ direction using a $20 \times 20$ lattice, $\mathrm{U} / \mathrm{t}=6,\langle\mathrm{n}\rangle=0.9$ for $\mathrm{T} / \mathrm{t}=0,1 / 50,1 / 20,1 / 6$ and $1 / 4$.

(b) Mean field determination of the region where pockets, define as a relative minimum along the $\mathrm{k}_{\mathrm{x}}=\mathrm{k}_{\mathrm{y}}$ direction, are observed in the $\mathrm{U} / \mathrm{t}$ vs $\mathrm{T} / \mathrm{t}$ plane for $\langle\mathrm{n}\rangle=0.9$ on a $20 \times 20$ cluster. The crosses indicate the lowest temperatures that can be reached with our Monte Carlo technique for different values of the coupling, due to the sign problem. 
4. (a) Momentum distribution $\mathrm{n}(\mathbf{k})$ as a function of momenta along the $(\pi, 0)$ to $(0, \pi)$ direction on an $8 \times 8$ cluster for $\mathrm{U} / \mathrm{t}=6, \mathrm{~T}=\mathrm{t} / 4$ at $\langle\mathrm{n}\rangle=1$ (filled circles), $\langle\mathrm{n}\rangle=0.9$ (open circles) and $\langle\mathrm{n}\rangle=0.75$ (filled triangles).

(b) Same as (a) for $\mathrm{U} / \mathrm{t}=4$.

(c) Momentum distribution $\mathrm{n}(\mathbf{k})$ as a function of momentum along the $(\pi, 0)$ to $(0, \pi)$ direction on an $8 \times 8$ lattice at $\langle\mathrm{n}\rangle=0.9$ and $\mathrm{T} / \mathrm{t}=1 / 4$ for (from above to below) $\mathrm{U} / \mathrm{t}=6,4,2$ and 0.

(d) Momentum distribution $\mathrm{n}(\mathbf{k})$ as a function of momentum along the $(\pi, 0)$ to $(0, \pi)$ direction on an $8 \times 8$ lattice at $\langle\mathrm{n}\rangle=0.9$ and $\mathrm{U} / \mathrm{t}=4$ for temperatures (from above to below) $\mathrm{T}=\mathrm{t} / 2, \mathrm{t} / 3, \mathrm{t} / 4$ and $\mathrm{t} / 6$. 